\title{
Purulent Pleurisy of Children: About 42 Cases in Senegal
}

\author{
Idrissa Basse ${ }^{1,}$ *, Ndiogou Seck ${ }^{3}$, Lamine Thiam ${ }^{4}$, Djibril Boiro ${ }^{2}$, Aliou Abdoulaye Ndongo ${ }^{5}$, \\ Amadou Sow ${ }^{2}$, Amadou Lamine Fall ${ }^{2}$, Moussa Cisse ${ }^{1}$, Ndieme Ndiaye ${ }^{1}$, Dina Obambi ${ }^{1}$, \\ Ndeye Ramatoulaye Diagne Gueye ${ }^{1}$, Ousmane Ndiaye ${ }^{2}$ \\ ${ }^{1}$ Pediatric Department, University of Thiès, Thies, Senegal \\ ${ }^{2}$ Pediatric Department, University of Dakar, Dakar, Senegal \\ ${ }^{3}$ Pediatric Department, University of Saint Louis, Saint Louis, Senegal \\ ${ }^{4}$ Pediatric Department, University of Ziguinchor, Ziguinchor, Senegal \\ ${ }^{5}$ Pediatric Department, Cheikh Anta Diop University of Dakar, Dakar, Senegal
}

Email address:

idrissabasse@yahoo.fr (I. Basse)

${ }^{*}$ Corresponding author

\section{To cite this article:}

Idrissa Basse, Ndiogou Seck, Lamine Thiam, Djibril Boiro, Aliou Abdoulaye Ndongo, Amadou Sow, Amadou Lamine Fall, Moussa Cisse, Ndieme Ndiaye, Dina Obambi, Ndeye Ramatoulaye Diagne Gueye, Ousmane Ndiaye. Purulent Pleurisy of Children: About 42 Cases in Senegal. International Journal of Infectious Diseases and Therapy. Vol. 6, No. 1, 2021, pp. 1-5. doi: 10.11648/j.ijidt.20210601.11

Received: November 25, 2020; Accepted: December 10, 2020; Published: January 4, 2021

\begin{abstract}
Introduction: Purulent pleurisy refers to the presence in the large pleural cavity of a purulent, cloudy or lemonyellow liquid, but containing altered polynuclear cells. It is a frequent pathology, the epidemiology of which is constantly changing in Africa and throughout the world. This is why we conducted this study with the aim of updating epidemiological, clinical, bacteriological and therapeutic data. Methods: This is a retrospective, mono-centric, descriptive study including the records of children aged 0 to 15 years old hospitalized during the period from 20 May 2014 to 26 March 2019 at the Children's Hospital of Diamniadio for purulent pleurisy. The data were entered and analyzed using sphinx, Excel under Windows 8 and SPSS software. Results: We counted 42 cases of purulent pleurisy, i.e. a hospital frequency of $0.7 \%$. Most of the patients were aged between 2 and 12 years (48\%). The average age at diagnosis was 46 months. The sex ratio was 2.5. Most of the children came from parents with a low socio-economic status (64\%); fever was the most frequent reason for consultation $(50 \%)$, followed by cough (33\%) and respiratory difficulty $(29 \%)$. In all cases, a liquid effusion syndrome was objectively diagnosed $(100 \%)$, tachycardia in $36 \%$ of cases and a pulmonary condensation syndrome in $33 \%$ of patients. X-rays showed a predominantly right-sided effusion $(62 \%)$ which was very abundant in $71 \%$ of cases. In bacteriology, staphylococcus aureus was the most frequently isolated germ (75\%), followed by streptococcus pneumoniae (20\%) and group D streptococcus $(5 \%)$. Biology showed an average hemoglobin level of 9.2 and there was a predominantly neutrophilic hyperleukocytosis in $98 \%$ of cases. All patients had received antibiotic therapy and pleural drainage in $76 \%$ of cases. Mortality was $10 \%$. Conclusion: In the light of this work, we insist on the importance of early diagnosis and adequate management of purulent pleurisy in order to avoid complications and after-effects. Thus, antibiotic therapy should be more closely monitored, and its administration better codified to reduce bacterial resistance and germ selection.
\end{abstract}

Keywords: Purulent Pleurisy, Children, Dakar

\section{Introduction}

Purulent pleurisy is a respiratory infection characterized by the presence of a purulent, squiggly or citrine liquid containing altered polynuclear cells in the large pleural cavity [1]. Acute respiratory infections (ARI) are the leading cause of morbidity and mortality in children under five years of age (5 years), especially in developing countries $[2,3]$. They are dominated by pneumonia, of which pleurisy is one of the major complications. Pneumonia is the second leading cause of infant and child mortality after neonatal mortality [4]. In Senegal, it accounts for $16 \%$ of mortality among children under 5 years of age [4]. Its association with pleurisy is even 
more serious. Since the 1990s, an increase in the incidence of purulent pleurisy has been observed in different countries around the world $[5,6]$. In Africa, some studies in hospitals report prevalence rates of $0.7 \%$ in Burkina Faso [7] and $0.9 \%$ in Morocco [8]. In Senegal, the Ndiaye study carried out at Dakar University Hospital noted a prevalence of $0.8 \%$ [9]. This resurgence is thought to be due on the one hand to the virulence and resistance of bacteria to antibiotics and on the other hand to factors specific to the host such as young age, malnutrition, immunosuppression and poor vaccination coverage. As a result, the epidemiology is constantly changing, which justifies this work whose objectives were: to describe the epidemiological profile, to determine the morbidity factors associated with purulent pleurisy, to identify the germs responsible and to assess the antibiotic treatments currently in use.

\section{Methods}

This is a retrospective, descriptive and analytical study, lasting 4 years and 10 months (from 20 May 2014 to 26 March 2019), carried out at the Children's Hospital of Diamniadio, which is a level III hospital. The study included all children aged 0-15 years admitted for pleurisy purulent pleurisy during the study period. The diagnosis of purulent pleurisy was based on purulent, cloudy or citrus-yellow puncture fluid containing altered neutrophil polynuclear and/or the presence of a pyogenic germ in the pleural fluid. For each patient, we noted the following data: Sociodemographic data, clinical data, paraclinical data and evolution. The assessment of the socioeconomic level (SEL) was based on the cumulative income of the parent (s) in relation to the GMIS (Guaranteed Minimum Interprofessional Salary) which is 80 dollars per month in Senegal. Thus, the SEL was considered low if this income was less than or equal to the GMIS, medium if it was higher than the GMIS but less than 5 times the GMIS, and good if the income was higher than 5 times the GMIS. The data was collected from the patients' files, hospitalization registers on a pre-established collection sheet. The data were entered and analyzed using sphinx software, Microsoft office Excel 8 under Windows 8 and SpSS for impedance testing. The quantitative variables were analyzed by determining the maximum, minimum, mean and standard deviation, while for the qualitative variables the frequency and percentage were determined.

\section{Results}

1- Epidemiology and sociodemographic: We collected 42 cases of purulent pleurisy with a hospital frequency of $0.7 \%$. The sex ratio was 2.5. The average age of our patients was 46 months and the age group 2 - 12 years was more represented (Figure 1) with $48 \%$ of the cases $(n=20)$.

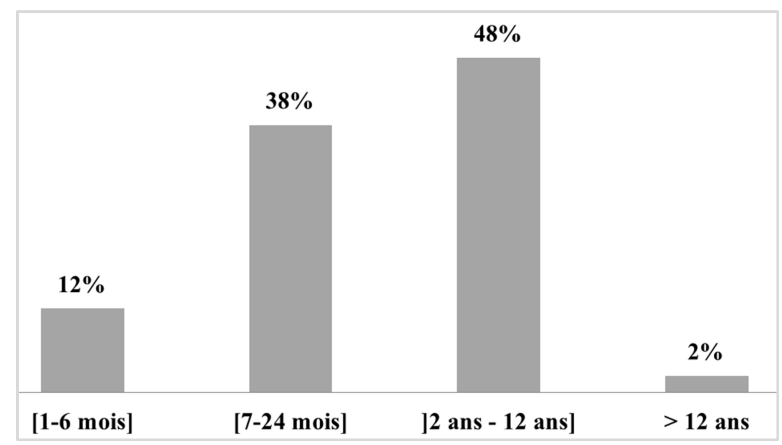

Figure 1. Distribution of patients by age group.

Most cases of purulent pleurisy occurred during the cooler period, with peaks in january, march, april and may. The year 2018 recorded a higher number of cases of purulent pleurisy with $28.57 \%(n=12)$. Most of the children came from the urban areas of Dakar (capital of Senegal). Sixty-four percent of them $(n=27)$ had a low socio-economic level. The vaccination was specified for 22 patients $(52 \%)$ and was complete for $21(96 \%)$.

2- Clinical data: The average delay between the onset of symptoms and consultation was 8 days with extremes of 1 and 30 days; the main reasons were fever 50\%, cough $33 \%$ and respiratory difficulty $29 \%$ (Figure 2). The functional and general manifestations present at admission were dominated by cough in $100 \%$ of cases, dyspnea in $93 \%$, fever in $50 \%$ of cases, chest pain in 36\%, and tachycardia in 36\%. Physical examination revealed mainly pleural fluid effusion syndrome in $100 \%$ of cases, respiratory distress in $93 \%$ of cases $(n=39)$, pulmonary condensation syndrome in $33 \% \quad(n=14)$. Otolaryngology damage was found in $17 \%$ of cases $(n=7)$. From the point of view of tolerance, severe hypoxia was found in 3 patients $(4.8 \%)$, and moderate hypoxia in 16 patients (38.1\%). Malnutrition, sickle cell disease and asthma were the main causes of purulent pleurisy.

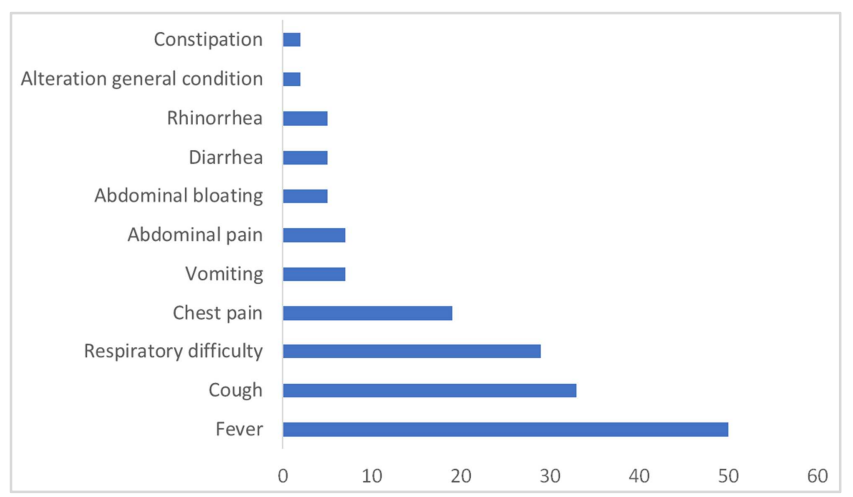

Figure 2. Distribution of patients by reason for consultation in percentage.

3- Complementary examinations: Chest X-ray showed bilateral effusion in all patients with a predominantly right-sided $62 \%(n=26)$. In addition to the effusion, pneumonia was present in $12 \%$ of cases $(n=5)$. Thoracic ultrasound was performed in 14 patients and noted a partition in 3 cases, and an encysting in 2 cases. A thoracic CT scan was performed in 7 patients and noted in addition to the effusion, pachypleuritis in 2 cases, 
pneumothorax in 2 cases and a cyst in 1 case.

Anemia was found in 40 patients $(95 \%)$. A predominantly neutrophilic hyperleukocytosis was noted in $98 \%$ of cases $(n=41)$, and thrombocytosis in $62 \%(n=26)$. The C-Reactive protein was positive in all our patients, with a mean of $158 \mathrm{mg} / \mathrm{l}$ with extremes of $12 \mathrm{mg} / 1$ and $384 \mathrm{mg} / \mathrm{l}$. Concerning the pleural fluid analysis: the effusion was exudative in all patients, with cellularity between 10 and 50 elements in $71 \%$ of patients $(n=30)$ with a predominance of altered neutrophil polynuclear cells. The bacteriological study isolated one bacterium for 20 cases with a positivity rate of $48 \%$ in the pleural fluid. Staphylococcus aureus was the first responsible germ in $35 \%$ of cases $(n=15)$, followed by streptococcus pneumoniae in $9 \%$ of cases $(n=4)$ and finally group $D$ streptococcus in $2 \%$ of cases $(n=1)$ (Figure 3$)$.

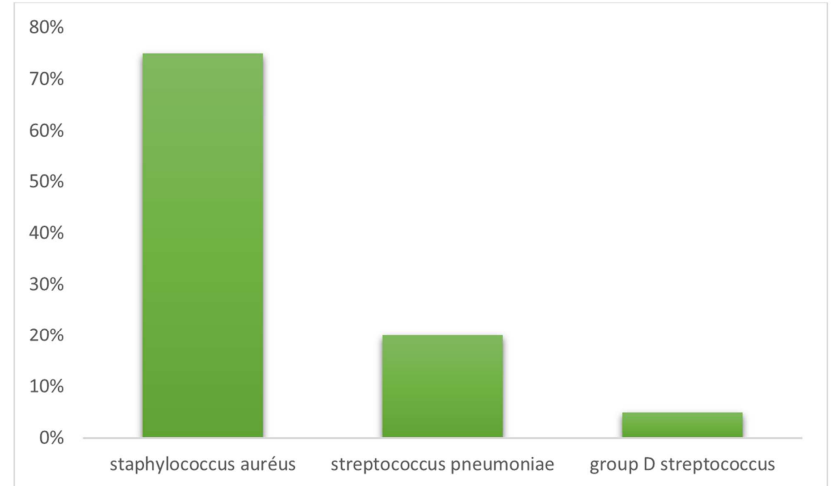

Figure 3. Distribution of patients by germ isolated in pleural fluid.

4- Treatment: Gentamicin was used in $67 \%$ of cases $(n=28)$, ceftriaxone in $64 \%$ of cases $(n=28)$, oxacillin in $55 \%$ of cases $(n=23)$, ampicillin in $29 \%$ of cases $(n=12)$. The average duration of intravenous antibiotic therapy was 21 days. Percutaneous pleural drainage was required in 32 patients $(76 \%)$.

5- Evolution: The average length of hospitalization was 20 days, with extremes of 1-42 days, with a favorable evolution for 38 patients (90\%). The main complications were septic shock $42 \%(n=18)$, empyema $17 \%(n=7)$, pneumothorax $17 \%$ $(n=7)$ and pachypleuritis $15 \%(n=6)$ (Table 1$)$. The lethality was $10 \%(n=4)$, including 3 deaths occurring beyond 72 hours of hospitalization.

Table 1. Distribution of patients according to complications.

\begin{tabular}{lll}
\hline Complications & Number & Percentage \\
\hline septic shock & 5 & $42 \%$ \\
enkystement & 5 & $42 \%$ \\
pleural empyema & 2 & $17 \%$ \\
pneumothorax & 2 & $17 \%$ \\
deshydration with shock & 1 & $8 \%$ \\
drain obstruction & 1 & $8 \%$ \\
Pachypleurite & 1 & $8 \%$ \\
Hypokaliemia & 1 & $8 \%$ \\
\hline
\end{tabular}

\section{Discussion}

During the twentieth century, the incidence of pleuropneumonia worldwide, particularly in developed countries, fell sharply, mainly due to advances in antibiotic therapy and improved hygiene conditions [10]. In Africa, the incidence in hospitals is still high, at around 0.7 and $0.9 \%$ respectively in Burkina Faso [7] and Morocco [8]. Similar incidences were found in another study in Senegal and in our own study with $0.8 \%$ by Ndiaye et al. in 1994 . As was the case in our work, purulent pleurisy can occur at any age, but infants and young children under five years of age are most affected [11]. The vulnerability of this age group is thought to be related to the immaturity of their immune system and the frequency of otorhinolaryngology and skin infections, which are the main entry points for severe respiratory infections [12]. Most studies report a male predominance without a rational explanation for this [13]. Families with a low socio-economic level have been highly represented in this work due to limited access to health services and probably less knowledge of basic preventive behaviors in addition to the often long therapeutic itinerary in our context. Thus, overcrowding and the nature of habitats are factors that favor acute respiratory infections. It should also be noted that, due to the geographical location of the structure that housed the study, most of our patients came from urban areas, where $67 \%$ of the cases were found to be in urban areas, where there is a deterioration in air quality with significant pollution peaks [14]. The limited resources in these areas, self-medication and recourse to traditional medicine were also the cause of significant delays in consultations, resulting in very abundant pleurisy pictures, which we found in most of our work (71\%). In $17 \%$ of our patients there was an otorhinolaryngology entrance door. Most cases of purulent pleurisy remain a complication of bronchopulmonary and otorhinolaryngological infections. Bacterial colonization and adhesion to the nasopharynx is promoted by viral infections, which subsequently induce bacterial diffusion to the lower airways [15]. These severe respiratory infections are also promoted by the existence of a weakened environment, which are important promoting factors [16]. In our work we found anemia, malnutrition and sickle-cell anemia. HIV infection is also a major contributor, but we had not found any cases of HIV infection. However, as the search for it is not systemic, we cannot exclude the existence of undetected subjects. At the time of the clinical examination, the main complaint associated with pleural involvement was pneumonia. This association is frequent because of the anatomical reports as reported in most studies [17]. From the point of view of additional examinations, the chest X-ray, which is essential before any pleurisy, most often allowed the diagnosis to be made. The profile $\mathrm{x}$-ray is useful to clarify the topography and the lateral decubitus front $\mathrm{x}$-rays on the side of the effusion (Muller's incidence) help to visualize a low abundance effusion. We found a predominantly right-sided effusion ( $62 \%$ versus $38 \%$ on the left). This righthand predominance has also been reported by several authors and could be explained mainly by the anatomical arrangement of the bronchi [9, 7, 8]. Ultrasound, in addition to X-ray images, is a valuable aid to management, as it allows guided echo punctures in the case of localized pleurisy, but also to establish the indication for surgical treatment in the case of 
multiple septal defects [18]. Classically, the cytochemical examination allows the therapeutic indication to be established, but in certain cases the lack of means of exploration in our context reduces the importance of the contribution of this examination. The bacteriological diagnosis of the child's pleurisy is of paramount importance. It allows epidemiological surveillance and guides antibiotic therapy, especially in a context where bacterial resistance is becoming more and more frequent. A bacterium was isolated from the pleural fluid in 20 cases (48\%) in our series. This low bacteriological yield could be explained by taking antibiotics before the pleural puncture. New techniques such as Polymerase chain reaction (PCR) could improve sensitivity as is the case in developed countries [13]. Staphylococcus aureus was the main germ responsible with $35.71 \%$, which was the case in a previous study carried out in DAKAR in 1994 (50.7\%) [9]. On the other hand, El Omrani [19] found streptococcus pneumoniae as the predominant germ. The serotyping of pneumococcal strains isolated in our work $(9.52 \%)$ was not available for comparison with the vaccine serotypes contained in the vaccine used in the Senegalese vaccination program. This is a limitation of our work. Therapeutically, antibiotic therapy has remained classical with ceftriaxone and gentamicin or oxacillinceftriaxone-gentamicin as the first line of treatment, secondarily adapted to the antibiogram. This therapeutic choice has always been adopted by Dakar prescribers for years, as shown by the work of NDIAYE in 1994 [9]. In our patients, the average duration of antibiotic therapy was 21 days, the duration recommended by the World Health organization [20]. The aim of corticosteroid therapy is to reduce pleural inflammation and consequently to limit the thickening of the pleural layers, thus improving the functional prognosis. In Dakar, we noted that its use decreased from $17.9 \%$ to $6.97 \%$ in 2007 and 2013 respectively, $[9,19]$ to a much lower rate in our study of $4.76 \%$. In the work of NDIAYE, it was shown that patients who received corticosteroids had no benefit compared to children who did not receive them [9]. The LARROQUET team in France recommends pleural evacuator puncture as a first-line technique for uncomplicated effusions (no septum) [10] However, in the Anglo-Saxon management guide, the use of repeated evacuator punctures is not recommended, and the authors prefer the use of a thoracic drain [8]. In our study, only 1 patient had benefited from a pleural evacuator puncture and 32 patients had percutaneous chest drainage (76\%). The indication for pleural drainage remains controversial. For some authors it should be undertaken at the beginning of the collection, and the results can be excellent, as in the DEBESSE series [21], where healing was obtained in $100 \%$ of cases. The results become less good when the drain is placed late, as in the HASSAN series with $64 \%$ healing [22]. Sometimes thoracic drainage is not able to dry out the effusions and avoid after-effects, in which case other therapeutic modalities such as surgery and the use of fibrinolytics can be used [22, 8]. In general, the lethality of purulent pleurisy from all causes is low. Indeed, in our series we observed a mortality rate of $10 \%$, higher than that reported by NDIAYE (6.5\%) [9] in 1994 and EL Omrani (3.87\%) in 2013 [19], although we have no explanation for this, although antibiotic resistance and hospital superinfections could be possible. Even higher mortality rates have been reported by other authors: $21.4 \%$ Bekri [23], 27.8\% SAWAGOGO [24].

\section{Conclusion}

This study shows that purulent pleurisy in children is still a frequent reason for hospitalization with significant lethality. Despite these results, we believe that prospective multicenter studies should be conducted to have nationally representative frequency and distribution. The prognosis could be improved thanks to the early recognition of the signs and to adapted prevention measures but also by a therapeutic strategy, above all symptomatic, rapid and based on protocols that will have to be established.

\section{References}

[1] Ferre A, Dres M, Azarian A. Purulent pleurisy. EMC pulmonology 2011; 6-041-A-40.

[2] Integrated management of childhood illness. A WHO/UNICEF initiative Bull World Health Organ 1997; 75 (Suppl 1) (31p).

[3] OMS. Statistiques sanitaires mondiales 2012. Organisation mondiale de la santé (OMS) 2012: 176p.

[4] Organisation mondiale de la santé (OMS). World Health statistics 2009 [online]. Disponible sur: www.who.Int/whosis/whostat/2009/en/index/htlm. Consulté le 24 octobre 2019 .

[5] Byington C, Spencer 1, Johnson T et al. An epidemiological Investigation of a sustained high rate of pediatric parapneumonic empyema: risk factors and microbiological associations. Clin Fac Dis 2002; 34: 434-40.

[6] Eastham KM, Freeman R, Kearns AM et al. Clinical features etiology and outcome of empyema in children in the north east of England. Thorax 2004; 59: 522-525.

[7] Koueta F, Ouedraogo SO, Ouedraogo G et al. Childhood pleurisy: epidemiological, clinical, para-clinical, therapeutic and progressive aspects at the pediatric university hospital Carles De Gaulle de Ouagadougou (Burkina Fasso). Clinics in Mother and Child Health 2011; 8: 6 p.

[8] Zeriouel A. Purulent pleurisy in children (about 35 cases). Méd Thesis, Fès, 2010; ํo25.

[9] Ndiaye O. Purulent pleurisy caused by banal germs in children. Thèse Méd., Dakar, 1990; No. 76.

[10] Blanc P, Dubus JC, Bosdure E, and all. Communal purulent pleurisy in children. Where are we?. Arch Pédiatr 2007; 14: 64-72.

[11] Amon-Tanoh-Dick F, Timite-Konan AM, Biafry MM and al. Non-tuberculous bacterial pleuro-pneumonitis in children in Abidjan. Health Notebooks; 199; 9: 145-9.

[12] Guyon G, Allal H, Lalande M and al. Purulent pleurisy in children: the Montpellier experience. Arch Pédiatr 2005; 12: s54-s57. 
[13] Weil-olivier C, Levy C, Marguet C, and al. Multicenter retrospective survey on infectious pleuropneumopathies in children in France. Arch Pédiatr 2005; 12: 823-826.

[14] Ndiaye T. Impact of particulate air pollution (PM10 and PM2.5) on the occurrence of asthma attacks in children at the Albert Royer National Children's Hospital (CHNEAR) in Fann Dakar. Thesis Med., University Cheikh Anta DIOP, Dakar, 2018, $\mathrm{N}^{\circ} 153$.

[15] Dagnra AY, Awesso B, Prince-David M and al. Nature and sensitivity to antibiotics of bacteria isolated from purulent pleurisy in Lomé (Togo). Med Mal Infect 2003; 33: 327-330.

[16] Kane M. Invasive pneumococcal infections at the Albert Royer National Children's Hospital in Dakar: about 218 cases collected from January 1, 2008 to December 31, 2013. Thesis Med., University Cheikh Anta DIOP, Dakar, 2014, N 60.

[17] Thumerelle C, Santos C, Morillon S et al. Risk factors for the occurrence of bacterial pleuro-pneumonia in pediatrics. Arch Pédiatr 2005; 12: 827-829.

[18] Grevent D, Ledosseur P, Cellier C et al. Treatment of purulent pleurisy in children: what to expect from imaging. Journal of Radiology 2004; Vol 85, Issue 9: P 1564.
[19] EL Ormani A. Purulent pleurisy caused by banal germs in children at the Albert Royer National Children's Hospital in Dakar: about 129 cases collected from January 1, 2007 to June 3, 2012. Thesis Med., Université Cheikh Anta DIOP, Dakar, $2013 ; \mathrm{N}^{\circ} 18$.

[20] Mccallum A, Baranwal AK. What are appropriate empiric antibiotics for empyema? International Child Health Review Collaboration, p. 1-6 www.ichrc.org, consulté le 19/03/2019.

[21] Dabesse B, Bellemy J. Pleural drainage and eradication of the pulmonary focus: standard treatment for acute purulent pleurisy with banal germs. Rev Fr Mal Resp 1983; 11: 245246.

[22] Hassan I, Mabogunye O. Adult empyeama in Zaria, Nigeria. East Afr Med J 1992; 69: 97-100.

[23] Bekri H, Cohen R, Varon $\mathrm{E}$ et al. Distributions of pneumococcal serotypes responsible for childhood pleuropneumopathies in France. Arch Pédiatr 2007; 14: 239243.

[24] Sawadogo A, Koweta F, Sanou I and al. Pleura-pulmonary staphylococcal disease in infants in a hospital environment in Ouagadougou (Burkina Fasso). Health Notebooks, 1999; 9: 209-13. 